\title{
Correction to: The TANDEM investigation: efficacy and tolerability of levodopa-carbidopa intestinal gel in (LCIG) advanced Parkinson's disease patients
}

\author{
Angelo Antonini ${ }^{1}$. Giovanni Abbruzzese ${ }^{2} \cdot$ Alfredo Berardelli $^{3,4} \cdot$ Nicola Modugno $^{5} \cdot$ Italo Stroppa $^{6}$. \\ Filippo Tamma ${ }^{7} \cdot$ Mariachiara Sensi $^{8} \cdot$ Francesca Mancini $^{9} \cdot$ Giovanni Cossu $^{10} \cdot$ Alessandro Stefani $^{11}$. \\ Nicola Tambasco ${ }^{12}$. Alessandro Tessitore ${ }^{13}$. Giovanni Fabbrini ${ }^{3,4}$. Francesco E. Pontieri ${ }^{14}$. Paolo Solla ${ }^{15}$. \\ Anna Rita Bentivoglio ${ }^{16,17}$. Cristoforo Comi ${ }^{18}$. Brigida Minafra ${ }^{19} \cdot$ Giulio Riboldazzi $^{20}$ • Donato Melchionda ${ }^{21}$. \\ Tommaso Martino ${ }^{21} \cdot$ Leonardo Lopiano ${ }^{22}$
}

Published online: 3 June 2020

○) Springer-Verlag GmbH Austria, part of Springer Nature 2020

\section{Correction to: Journal of Neural Transmission https://doi.org/10.1007/s00702-020-02175-1}

The original version of this article unfortunately contained a mistake. Alfredo Berardelli and Giovanni Fabbrini affiliations were incorrect.

The original article can be found online at https://doi.org/10.1007/ s00702-020-02175-1.

\section{Angelo Antonini}

angelo3000@yahoo.com

1 Parkinson and Movement Disorders Unit, Department of Neuroscience, University of Padua, Padua, Italy

2 Department of Neuroscience, Rehabilitation, Ophthalmology, Genetics and Maternal Child Health, University of Genoa-IRCCS Ospedale Policlinico San Martino, Genova, Italy

3 Department of Human Neurosciences, Sapienza University of Rome, Rome, Italy

4 IRCCS Neuromed, Pozzilli, Isernia, Italy

5 Parkinson Centre-IRCCS Neuromed, Pozzilli, Isernia, Italy

6 U.O. Dipartimentale di Endoscopia Digestiva Operativa e D’Urgenza, Policlinico Tor Vergata, Rome, Italy

7 Neurology Unit, Miulli Hospital, Acquaviva delle Fonti, BA, Italy

8 Neurology Unit, Hospital Sant'Anna, Cona, Ferrara, Italy

9 UO Neurologia e Stroke Unit e Laboratorio Neuroscienze, Istituto Auxologico San Luca, Milan, Italy

10 SC Neurologia e Stroke Unit, Azienda Ospedaliera Brotzu, Cagliari, Italy

11 UOSD Parkinson, Fondazione Policlinico Tor Vergata, Rome, Italy
The correct affiliation of Alfredo Berardelli and Giovanni Fabbrini and author group are given.

In Table 1 there is a little mistake. The mistake is included in the part of the table describing the "Reason to start LCIG". The corrected Table 1 is placed in the following page.

12 Neurology Department, Perugia General Hospital and University of Perugia, Perugia, Italy

13 Department of Medical, Surgical, Neurological, Metabolic and Aging Sciences, University of Campania, "Luigi Vanvitelli", Naples, Italy

14 Department NESMOS, "Sapienza" University, Sant'Andrea Hospital, Rome, Italy

15 Neurology Unit, Policlinico Universitario Monserrato, Cagliari, Italy

16 Institute of Neurology, Università Cattolica del Sacro Cuore, Rome, Italy

17 Fondazione Policlinico Universitario A. Gemelli IRCCS, Rome, Italy

18 Parkinson's Disease and Movement Disorders Centre, Neurology Unit, University of Piemonte Orientale, Novara, Italy

19 Parkinson's Disease and Movement Disorders Unit, IRCCS C. Mondino Foundation, Pavia, Italy

20 Parkinson's Disease and Movement Disorders Centre, Neurology Unit, ASST dei Sette Laghi, Varese, Italy

21 S.C. Neurology, Neuroscience Department, A.O.U. Ospedali Riuniti, University of Foggia, Foggia, Italy

22 Department of Neuroscience "Rita Levi-Montalcini", University of Torino, Torino, Italy 
Table 1 Demographics, medical history, PD characteristics, symptoms, motor fluctuations, complications and quality of life (QoL) recorded at $\mathrm{BL}$ $[$ mean $\pm \mathrm{SD}, N=(\%)$ ]
Gender

Male

$88(55 \%)$

Female

$71(45 \%)$

Age (years)

$69.1 \pm 6.6$

Weight $(\mathrm{kg})$

$70.0 \pm 12.5$

BMI

$25.3 \pm 3.6$

Medical history

Cognitive decline (mild/moderate)

$52(33 \%)$

Psychosis

$7(5 \%)$

Depression

Compulsive disorder

$44(28 \%)$

$7(5 \%)$

Dementia

$5(3 \%)$

Family history

Parkinson disease

$86(54 \%)$

Parkinsonism

$2(1 \%)$

Tremor

$8(5 \%)$

Other Parkinson syndromes

$8(5 \%)$

Dementia

$8(5 \%)$

PD characteristics

Time since PD diagnosis (years)

$13.6 \pm 5.6$

Time since fluctuations (years)

$8.4 \pm 4.0$

Previous PD treatment

Levodopa

$98 \%$

Dopamine agonist

$79 \%$

$54 \%$

$41 \%$

$21 \%$

$5 \%$

$3 \%$

$5 \%$

Other oral

Severity stage

Hoehn and Yahr "On"

$2.6 \pm 0.7$

Hoehn and Yahr "OFF"

$3.6 \pm 0.8$

PD symptoms

Tremor [unilateral/bilateral]

Rigidity [unilateral/bilateral]

Bradykinesia [unilateral/bilateral]

Postural instability

Hyposmia

Anxiety

Depression

Apathy

Others $^{\mathrm{a}}$

$90(57 \%)[83(52 \%) / 7(4 \%)]$

97 (61\%) [83 (52\%)/14 (9\%)]

$111(70 \%)[80(52 \%) / 31(20 \%)]$

$31(20 \%)$

$11(7 \%)$

$13(8 \%)$

$31(20 \%)$

$6(4 \%)$

$15(9 \%)$

Complications

Pain

$63(42 \%)$

$85(5 \%)$

Freezing

$48(32 \%)$

Dystonia

$72(47 \%)$

Fall

$96(63 \%)$

Motor fluctuations

UDDRS Part III total score in "On" $(N=140)$

$28.0 \pm 13.3$

UDDRS Part III total score in "OFF" $(N=131)$
$45.8 \pm 13.2$ 
Table 1 (continued)
Complications of therapy
UPDRS part-IV total score $(N=133)$
$8.8 \pm 2.9$
Quality of life
PDQ-8 total score $(N=61)$
$17.0 \pm 8.0$
$\operatorname{ADL}(N=62)$
$4.6 \pm 3.4$
PDQ-39 total score $(N=39)$
$36.7 \pm 20.2$
Reason to start LCIG
Motor fluctuations
$143(90 \%)$
Dyskinesia
$86(54 \%)$
Prolonged OFF
$105(66 \%)$
Others ${ }^{\mathrm{b}}$
$13(8 \%)$

TANDEM population $(N=159)$

Number of observations in efficacy assessments: UPDRS part-III "On" $(\mathrm{N}=140)$, UPDRS part-III "OFF" $(N=131)$, UPDRS part-IV $(N=133)$, PDQ-8 $(N=61)$, ADL $(N=62)$ and PDQ-38 $(N=39)$

$P D$ Parkinson's disease, $A D L$ activities of daily living, $Q o L$ quality of life, UPDRS Unified Parkinson's Disease Rating Scale, $P D Q-8$ 8-item Parkinson's Disease Questionnaire, $P D Q$-39 39-item Parkinson's Disease Questionnaire, $D B S$-STN deep brain stimulation of the subthalamic nucleus, $S c$ subcutaneous, COMT catechol- $O$-methyltransferase, $M A O-B$ monoamine oxidase

${ }^{a}$ Asthenia, headache, pain, gait disturbances, visual disturbances, hypophonia

${ }^{b}$ Deep brain stimulation of the subthalamic nucleus, non-motor OFF, blepharospasm, dysphagia, dystonia, freezing, frequent falls

Publisher's Note Springer Nature remains neutral with regard to jurisdictional claims in published maps and institutional affiliations. 